\title{
Efectos de dosis bajas de alcohol sobre la memoria declarativa en humanos"
}

\section{Effects of low doses of alcohol on declarative memory in humans}

Recibido: enero 28 de 2010 | Revisado: mayo 14 de 2010 | Aceptado: junio 2 de 2010

\author{
José ARTURo BRÍNẼE-HORTA ** \\ RAÚl OYUELA-VARGAS \\ Pontificia Universidad Javeriana, Bogotá, Colombia
}

SICI: 1657-9267(201112)10:3<923:EDBAMD>2.3.TX;2-B

Para citar este artículo. Bríñez-Horta, J. A. \& Oyuela-Vargas, R. (2011). Efectos de dosis bajas de alcohol sobre la memoria declarativa en humanos. Universitas Psychologica, 10 (3), 923-935.

Agradecimientos especiales a Ana María Monroy, Luisa Merlano, María Claudia Cortés, estudiantes de Psicología y asistentes de investigación, y a Harold Guzmán, asistente del laboratorio.

*** Pontificia Universidad Javeriana, Bogotá. Tel. 3208320 ext. 5711. E-mail: jbrinez@javeriana. edu.co
RESUMEN

Se evaluó el efecto de 0, 0.035, 0.150, $0.300 \mathrm{~g}$ de alcohol sobre 2 componentes de la memoria explícita o declarativa, en 16 participantes, hombres y mujeres, mediante la aplicación de la prueba de textos del Weschler-III (WMS-III), utilizando un diseño factorial $2 \times 4$ de medidas repetidas contrabalanceadas. No se encontraron diferencias entre los sexos, pero sí entre las dosis, siendo $0.150 \mathrm{~g}$ de alcohol $/ \mathrm{kg}$ de peso corporal la que más decrementó el recuerdo de episodios y de temas, especialmente a mediano plazo, con tamaños de los efectos de 43.9 y $62.9 \%$, respectivamente. Estos resultados confirman que el alcohol, aun en dosis bajas, deteriora más el componente semántico del recuerdo explícito, aproximadamente media hora después de su consumo.

Palabras clave autores

Alcohol, dosis bajas, memoria declarativa, WMS-III.

Palabras clave descriptores

Investigación cuantitativa, consumo de sustancias psicoactivas, estudio experimental, procesos cognitivos.

\section{A B S T R A C T}

This study assessed the effect of low doses of alcohol $(0,0.035,0.150,0.300$ g) on two elements of explicit or declarative memory, in 16 participants, 8 women and 8 men, with The Weschler Memory Scale III Text Test. A factorial $2 * 4$ counterbalanced with repeated measures design was used. There were no statistically significant differences by gender, but there were differences among doses, specially $0.150 \mathrm{~g} / \mathrm{kg}$., which reduced episodic and semantic retrieval, between 43.9 and $62.9 \%$ of effect strength, in intermediate term memory. These results provided evidence that alcohol in low doses has a more pronounced effect in semantic, rather than episodic memory, in the middle term.

Key words authors

Alcohol, declarative memory, low doses, WMS-III.

Key words plus

quantitative research, psychoactive substance use, experimental desing, cognitive process. 
El alcohol, dependiendo de las dosis o del nivel de concentración de alcoholemia, altera la formación de la memoria explícita o declarativa a largo plazo, dificulta el aprendizaje que se adquiere inmediatamente después de su consumo y, en algunas ocasiones, produce pérdida de la información recibida mientras de ingiere. Sin embargo, dosis bajas de esta sustancia facilitan algunas tareas de vigilancia. Esta información, aún teórica y empíricamente poco consistente, dificulta la comprensión de los efectos del alcohol sobre los procesos cognitivos.

La memoria, por otra parte, es un conjunto de procesos cognitivos fundamentales en la vida de los organismos. Los habilita para adquirir, almacenar, recordar y modificar el conocimiento, para cambiar sus conductas de acuerdo con sus experiencias y para planear acciones futuras. La investigación psicológica y neurofisiológica ha permitido diferenciar estos procesos, en memorias a corto, intermedio y largo plazo; en memoria implícita: representaciones perceptuales y motoras o de procedimiento; en memorias explícitas o declarativas: episódica, similar a la episódica y semántica; en memoria espacial y en memoria de trabajo (Baddeley, 2000). Estas clasificaciones corresponden a diferentes sistemas, cada uno de los cuales tiene su paralelo neurofisiológico, consistente en redes neuronales semi-independientes, cuyas funciones se expresan en las conductas de los organismos (Wang \& Morris, 2010). El estudio de estos sistemas ha permitido evidenciar un conjunto de interconexiones neuronales hipocampo-corticales y desarrollar una teoría de la formación de la memoria que puede resumirse en 4 componentes, relacionados con las fases de codificación, consolidación, recuerdo y reconsolidación. En cada una de estas fases los organismos reciben información de objetos y eventos, y establecen relaciones entre ellos y con el lugar y el momento en que se perciben. Cuando esta información se recibe y se recuerda intencional y conscientemente, recibe el nombre de memoria declarativa o explícita (Eichenbaum, 2001); cuando no, se la denomina implícita (Kandel, 2001; Squire \& Zola, 1996). La memoria implícita se desarrolla de manera automática, sin intención y es difícil de modificar. La memoria declarativa, por el contrario, requiere de atención y de niveles altos de conciencia, se desarrolla lentamente e involucra síntesis de representaciones de episodios y eventos dentro de estructuras de conocimiento semántico. Se la ha subclasificado, en consecuencia, en memoria semántica y episódica, dependiendo de las relaciones de asociación que el organismo establezca entre los objetos, los hechos y el lugar o el tiempo en que ocurren (Tulving, 2002). Cuando esta última subcategoría no comprende la presencia o la acción del self se le denomina similar a la episódica (episodic-like) y se la considera propia de los organismos no humanos. Como tal, la memoria episódica puede ser implícita o explícita.

Estas características operativas -psicológicastienen sus paralelos neurales, fisiológicos y estructurales, distintos en cada fase de desarrollo de la memoria, formados por procesos sinápticos y circuitos neurales, que integran funciones de la Formación Hipocampal (FH) y sistemas sub y neocorticales:

- En la fase de codificación, en la que se recibe información y se forman representaciones de eventos nuevos localizados espacialmente, son cruciales a) la excitación de la capa III de la corteza entorrinal, b) la excitación de las células CA3, c) la neuromodulación dopaminérgica del Área Tegmental Ventral, d) la acción glutamatérgica y e) la inhibición reguladora, que se transmiten a las células CA1 del hipocampo (Wang \& Morris, 2010).

- En la fase de consolidación se selecciona la información y se determina el nivel de persistencia de los eventos seleccionados. Entre los factores psicológicos de la consolidación están: la asociación por cercanía temporal o espacial, la interferencia de contenidos de información, la novedad, el significado emocional, la relevancia de los eventos y la presencia de otros eventos que ocurren en el mismo contexto. Paralelamente a estos procesos psicológicos, ocurren: a) la síntesis y captura de nuevas proteínas, que estabilizan las huellas de memoria dentro de las neuronas, involucrando protein-kinasas que facilitan el transporte de mRNA y sus productos 
a los somas y dendritas (consolidación celular) y b) interacciones hipocampo-neocorticales (consolidación sistémica) (Wang \& Morris, 2010).

- El recuerdo se refiere a la recuperación de sistemas de información selectivos y parece que ocurre paralelo a la asociación entre áreas corticales e hipocampo, cuyas redes parecen estar asociadas a la rapidez con que se recuperan las memorias (Wang \& Morris, 2010).

- La reconsolidación ocurre posiblemente por comparación entre las situaciones actuales y experiencias anteriores, que desestabilizan las huellas almacenadas y las fortalecen o reorganizan mediante la incorporación de información nueva. Este concepto supone que las huellas de memoria consolidadas no son estables permanentemente; pueden ser inhibidas, modificadas, actualizadas, como ocurre, por ejemplo, en la inducción de amnesia o en las terapias para suprimir miedos y conductas de evitación condicionados a contextos. Se considera que la inhibición de la síntesis de proteínas puede ser uno de los mecanismos neurofisiológicos paralelos a estas expresiones conductuales (Suzuki et al., 2004).

La observación de estas expresiones conductuales ha sido una de las principales estrategias psicológicas para el estudio de la memoria. La identificación del paralelismo entre los mecanismos neurales y la memoria se ha basado en la descripción de los efectos de la alteración de la neurofisiología cerebral. Esta estrategia ha permitido identificar y describir los diversos sistemas de memoria y las fases de su desarrollo. Se producen lesiones neuroanatómicas o se altera la fisiología de los sistemas neurales y se observan los cambios conductuales. En los últimos años, sin embargo, se vienen utilizando sustancias que afectan farmacológica y selectivamente los sistemas neurofisiológicos que han mostrado paralelismo con las manifestaciones de la memoria. Una de estas sustancias, quizá la más común hoy día, es el alcohol. Su administración aguda traspasa rápidamente la barrera hematoencefálica y modifica en muy pocos minutos, entre 8 y12, de manera selectiva, las estructuras y circuitos que han mostrado constituir los diversos sistemas neurales donde se desarrollan las diferentes categorías de la memoria, más específicamente las memorias declarativa (Kirchner \& Sayette, 2003; Ray, Bates \& Bly, 2004), la espacial y la no espacial (Matthews \& Silvers, 2004).

El alcohol (etanol) es un compuesto orgánico de cadena corta, con fórmula $\mathrm{C}_{2} \mathrm{H}_{5} \mathrm{OH}$, soluble en lípidos, cuyo mecanismo de acción se consideró inicialmente como "difuso" en las membranas celulares. Sin embargo, hoy se considera que actúa específicamente en algunas áreas cerebrales, alterando sistemas neuroquímicos particulares, como el gabaérgico y el glutamatérgico. Su administración aguda potencia los mecanismos de inhibición mediados por los receptores $\mathrm{GABA}_{\mathrm{A}}$, aun en dosis bajas (Wallner, Hanchar \& Olsen, 2006), bloqueando los $\mathrm{GABA}_{\mathrm{B}}$ (Wan, Berton, Madamba, Francesconi \& Siggins, 1996) en la neuronas piramidales del hipocampo y bloquea, también, la inducción de la potenciación a largo plazo (PLP), dependiente del N-metil- d-aspartato (NMDA) (Blitzer, Gil \& Landau, 1990). Estos mecanismos de acción del etanol en el hipocampo están asociados con los mecanismos de acción que ocurren en los sistemas de memoria.

El concepto de dosis bajas, por otro lado, se refiere a cantidades de sustancia que no producen intoxicación aparente, según los criterios aceptados por las organizaciones internacionales que regulan el uso de sustancias. En relación con el etanol, hacen referencia al consumo o administración de soluciones menores o iguales al $10 \% \mathrm{v} / \mathrm{v}$ de alcohol absoluto, equivalentes a alcoholemias aproximadas a $1.7 \mathrm{mg} / \mathrm{dl}$ y que se obtienen ingiriendo $0.380 \mathrm{~g}$ de alcohol/kg de peso corporal, o menos, alrededor de 3 tragos estándar (Zwerling \& Jones, 1999).

La revisión presentada en esta introducción ha estado enmarcada en un conjunto de hipótesis, todas sustentadas ya empíricamente en los resultados de la investigación experimental.

1) En relación con el aprendizaje y la memoria, la hipótesis del mapeo cognitivo predice: a) que los animales tienen tendencia a procesar 
y utilizar la información espacial; b) que las tareas que se aprenden con fundamento en la información espacial se aprenden más rápidamente que las sustentadas en la no espacial; c) que cuando los animales pueden aprender espacial o no espacialmente, prefieren hacerlo espacialmente; y d) que el hipocampo es crítico para el aprendizaje espacial. Además, e) cuando el hipocampo es disfuncional, los animales aprenden utilizando información no espacial (Matthews \& Silvers, 2004).

2) En relación con los mecanismos neurales de la memoria, se tienen las hipótesis de: a) plasticidad sináptica, b) almacenamiento de la información seleccionada por mecanismos génicos, que estabilizan los cambios sinápticos y c) los sistemas de consolidación, formados por interacciones entre la neocorteza y el hipocampo, que predicen modificaciones de las memorias consolidadas por obtención de nueva información (Wang \& Morris, 2010).

3) En relación con el alcohol, se parte de la hipótesis de que el consumo agudo del alcohol, aun en dosis bajas, actúa de manera selectiva sobre algunos mecanismos neurofisiológicos, específicos del hipocampo, de tal manera que se puede utilizar provechosamente el modelo experimental no invasivo para el estudio de la memoria.

Este grupo de hipótesis constituye el conjunto de elementos que estructuró el modelo teórico, fundamento esta investigación. Aunque este modelo permite examinar tanto la memoria como el alcohol, el objetivo de la investigación fue estudiar los efectos de dosis bajas de etanol sobre algunos contenidos de la memoria declarativa, en hombres y mujeres. Se propusieron dos hipótesis: 1) dado que el alcohol no se metaboliza de igual manera en hombres y mujeres, se esperó que produjera efectos diferentes en los dos sexos y 2) que las diferentes dosis generaran efectos diferentes.

\section{Método}

\section{Participantes}

En este estudio participaron 8 hombres y 8 mujeres, entre 18 y 45 años de edad y 51 y $85 \mathrm{~kg}$ de peso corporal, con un Índice de Masa Corporal entre 18.37 y 29.89 y cuyo nivel de alcohol, medido en el aire pulmonar, varió entre 0 y 0.24 , con una media igual a 0.082 . Este número de participantes proporcionó 64 unidades de análisis, 16 x 4 condiciones experimentales, 32 correspondientes a los hombres y 32 a las mujeres. Fueron elegidos entre quienes recibieron información del estudio por parte del personal del Laboratorio de Psicología de la Facultad de Psicología de la Universidad Javeriana de Bogotá, Colombia, quienes aceptaron y ratificaron voluntariamente la invitación y satisficieron los criterios de inclusión y de exclusión preestablecidos. Algunos eran estudiantes de Psicología, otros, administradores de diversas dependencias de la universidad y otros más, familiares de los mismos participantes. Todos eran mayores de edad, según la legislación colombiana. Los criterios de exclusión fueron: 1) presencia de riesgos médicos, particularmente dificultades con el metabolismo de la glucosa, embarazo, consumo de medicamentos, alcoholismo familiar en línea vertical, historia de problemas neurológicos y uso actual de otras sustancias psicoactivas, especialmente consumo de bebidas alcohólicas más de 2 veces por semana, asociado a presencia de signos de dependencia o abuso alcohólicos; 2) presencia de riesgos psicológicos, tales como depresión, historia personal de tratamientos para desintoxicación farmacológica y observación de conductas de desadaptación social. Cada participante fue asignado aleatoriamente a una secuencia predeterminada de administración de las dosis de alcohol, de acuerdo con el diseño adoptado. Todos los participantes elegidos realizaron todas las pruebas, al final de las cuales recibieron un bono, de igual valor para todos, para ser cambiado por bienes en el mercado de la ciudad. 


\section{Diseño}

Se utilizó un diseño factorial $2 \times 4$, de medidas repetidas, con el sexo como factor intersujetos $y$ las dosis como factor intrasujeto, con 4 secuencias contrabalanceadas, cada una para 2 hombres y 2 mujeres, estructurado de la siguiente manera:

\begin{tabular}{cccccc}
\hline \multicolumn{2}{c}{ Sexo } & \multicolumn{5}{c}{ Dosis (Secuencia experimental) } \\
\hline $\begin{array}{c}\text { Hom- } \\
\text { bres }\end{array}$ & $\begin{array}{c}\text { Muje- } \\
\text { res }\end{array}$ & $\begin{array}{c}\text { Condi- } \\
\text { ción 1 }\end{array}$ & $\begin{array}{c}\text { Condi- } \\
\text { ción 2 }\end{array}$ & $\begin{array}{c}\text { Condi- } \\
\text { ción 3 }\end{array}$ & $\begin{array}{c}\text { Condi- } \\
\text { ción 4 }\end{array}$ \\
\hline 2 & 2 & $\begin{array}{c}\text { Control } \\
(0)\end{array}$ & 35 & 150 & 300 \\
2 & 2 & 35 & 300 & $\begin{array}{c}\text { Control } \\
(0)\end{array}$ & 150 \\
2 & 2 & 150 & $\begin{array}{c}\text { Control } \\
(0)\end{array}$ & 300 & 35 \\
2 & 2 & 300 & 150 & 35 & $\begin{array}{c}\text { Control } \\
(0)\end{array}$ \\
\hline
\end{tabular}

Nota. Las dosis están expresadas en mg de alcohol $/ \mathrm{kg}$ de peso corporal.

Se tuvieron, en consecuencia, 2 variables independientes, el sexo, con 2 niveles, y la dosis de alcohol, con 4 niveles: 0, 35, 150 y $300 \mathrm{mg}$ de alcohol por $1 \mathrm{~kg}$ de peso corporal. La variable dependiente, la memoria auditivo-verbal declarativa, se evaluó a corto y a mediano plazo, solicitándoles a los participantes que atendieran, para que recordaran aproximadamente 30 minutos después, 25 elementos o unidades conceptuales, agrupados en 7 temas.

\section{Instrumentos}

Para evaluar la memoria auditivo-verbal declarativa se utilizaron 4 conjuntos de 2 textos, un conjunto para cada condición experimental, 3 de ellos diseñados con contenidos diferentes, pero siguiendo la misma estructura del texto original de las escalas de la batería de memoria de Weschsler (WMS-III). Uno de los textos se utilizó para evaluar la memoria tanto a corto como a mediano plazo; el otro, evaluó solo la memoria a corto plazo. La versión inglesa de las escalas de esta batería posee un índice de confiabilidad entre 0.70 y 0.90; la versión española, entre 0.74 y 0.93 . Su coeficiente de estabilidad está entre el 0.50 y $100 \%$. Su validez concurrente varía entre 0.34 y 0.73 , cuando se ha comparado con el WMS-R, y entre 0.26 y 0.82 cuando la correlación se ha hecho con el WAIS-III (WMS-III, Manual teórico, 2004, pp. 57-87).

Para la administración de las dosis se utilizó etanol al $95 \%$, para consumo humano, adquirido en Rentas de Cundinamarca, siguiendo las normas estatales para su adquisición, y jugo de naranja natural. El nivel de alcohol se evaluó mediante un alcoholímetro profesional de precisión, digital, modelo CDP-6000.

\section{Procedimiento}

Los participantes se asignaron aleatoriamente a cada secuencia experimental antes de la implementación del estudio. Las dosis se prepararon inmediatamente antes de cada aplicación experimental, en ausencia de los participantes, de acuerdo con la siguiente fórmula:

(kg de peso corporal * gramos de etanol) / Densidad del alcohol $=\mathrm{ml}$ de alcohol absoluto

y se les añadió jugo de naranja natural hasta completar un coctel de $150 \mathrm{ml}$, que los participantes bebían en el curso de un período de 2 minutos. Inmediatamente después, el participante ingresaba a una cámara de Gesell, donde lo esperaba el investigador designado para manejar el software de presentación de las pruebas. Al cumplirse 12 minutos, se iniciaba la lectura de las instrucciones y de los textos, siguiendo el procedimiento del WMS-R. La filmación se realizaba dentro de la cámara de Gesell, se grababan las respuestas y se monitoreaba toda la sesión experimental en la sala de observación adyacente a la cámara experimental. No se realizó ningún registro manual durante la aplicación de las pruebas. Las instrucciones y los textos se presentaron electrónicamente, estandarizados, y la observación y el registro de las respuestas se realizaron posteriormente a su implementación, repasando las filmaciones realizadas. 
Las pruebas de memoria consistieron en la lectura de 2 textos que narraban 2 historias, cada una leída en 2 ocasiones. La historia 1 se leyó al principio y al final de la sesión experimental, con un intervalo de aproximadamente 30 minutos. Inmediatamente después de cada lectura, los participantes debían recordarla lo más exactamente posible. La historia 2 se leía en 2 ocasiones seguidas, inmediatamente después del primer recuerdo de la primera historia. Entre las lecturas de la primera historia se realizaron 2 pruebas adicionales que sirvieron como interferencia para el recuerdo a mediano plazo de la primera historia. Las sesiones experimentales tuvieron una duración entre 30 y 35 minutos, al final de las cuales se tomó 2 veces el registro del nivel de alcohol en aire pulmonar. Estas sesiones se realizaron 4 veces para cada sujeto, con dosis diferentes, en una secuencia diferente, según el diseño predefinido.

\section{Resultados}

Se evaluó el efecto de 3 dosis bajas de etanol, 0.035, 0.150 y 0.3 g de etanol sobre la memoria declarativa de 16 participantes, 8 hombres y 8 mujeres, entre 18 y 45 años de edad, de 51 y $81 \mathrm{~kg}$ de peso corporal y un Índice de Masa Corporal entre 18.37 y 29.89. Los participantes presentaron un promedio de concentración de alcohol, medida en el aire pulmonar, de 0.082 , con un rango entre 0 y 0.24 , tomadas todas las dosis en conjunto (Tabla 1). La concentración 0 correspondió a la dosis control.

\section{Datos generales}

La Tabla 2 muestra los rangos de variación de las concentraciones de alcohol que presentaron los participantes, en cada una de las dosis bajo las cuales realizaron las pruebas.

TABLA 2

Concentraciones pulmonares mínimas y máximas de alcohol, bajo cada una de las dosis, según el sexo

\begin{tabular}{lccc}
\hline \multirow{2}{*}{ Sexo } & \multicolumn{3}{c}{ Dosis de alcohol en gramos } \\
\cline { 2 - 4 } & 0.035 & 0.150 & 0.300 \\
\hline Hombres & 0.08 & $0.08-0.120$ & $0.08-0.150$ \\
Mujeres & 0.08 & $0.08-0.165$ & $0.09-0.240$ \\
\hline
\end{tabular}

Fuente: elaboración propia.

\section{Descriptivos}

Los descriptivos del recuerdo de las unidades de los textos a corto (MCP) y a mediano plazo (MMP), en cada sexo, se presentan en la Tabla 3. La Figura 1 ilustra los mismos resultados. Se observa, también, que el recuerdo a mediano plazo es inferior al recuerdo a corto plazo, bajo todas las dosis, incluyendo el control. Obsérvense, además los efectos característicos de 150 y de $300 \mathrm{mg}$ de alcohol.

\section{TABLA 1}

Datos generales de los participantes

\begin{tabular}{lcccccccc}
\hline \multirow{2}{*}{ Sexo } & \multicolumn{4}{c}{ Edad } & \multicolumn{5}{c}{ Peso corporal } \\
\cline { 2 - 9 } & Mín. & Máx. & Media & DT & Mín. & Máx. & Media & DT \\
\hline Hombres & 21 & 46 & 29.63 & 7.11 & 56 & 81 & 68.25 & 7.54 \\
Mujeres & 18 & 45 & 29.25 & 8.79 & 51 & 74 & 60.83 & 7.25 \\
\hline \multirow{3}{*}{} & \multicolumn{3}{c}{ İndice de Masa Corporal } & \multicolumn{4}{c}{ Alcohol en aire pulmonar } \\
\cline { 2 - 9 } & Mín. & Máx. & Media & DT & Mín. & Máx. & Media & DT \\
\hline Hombres & 18.37 & 27.08 & 23.71 & 2.75 & 0 & 0.15 & 0.08 & 0.044 \\
Mujeres & 21.72 & 29.01 & 24.89 & 2.51 & 0 & 0.24 & 0.083 & 0.087 \\
\hline
\end{tabular}

Nota. $N=16$. DT: Desviación Tipo.

Fuente: elaboración propia. 
TABLA 3

Descriptivos de la memoria declarativa , unidades de textos, según el sexo y la dosis

\begin{tabular}{llcccccccc}
\hline \multirow{2}{*}{ Sexo } & \multicolumn{8}{c}{ Dosis de alcohol en mg } \\
\cline { 3 - 10 } & & \multicolumn{2}{c}{0} & \multicolumn{3}{c}{35} & \multicolumn{2}{c}{150} & \multicolumn{2}{c}{300} \\
\hline \multirow{2}{*}{ Hombres } & Media & DT & Media & DT & Media & DT & Media & DT \\
& MCP & 44.75 & 8.63 & 40.83 & 10.78 & 30.88 & 7.2 & 39.38 & 10.08 \\
& MMP & 30.75 & 5.65 & 26.13 & 8.82 & 19.5 & 6.04 & 25.63 & 8.22 \\
& MCP & 45 & 7.03 & 43.75 & 7.59 & 31.5 & 9.05 & 41.88 & 8.57 \\
& MMP & 30.38 & 8.68 & 26.83 & 4.27 & 18.83 & 6.43 & 25.38 & 9.03 \\
\hline
\end{tabular}

Nota. $N=64$. La escala de medida hace referencia al número de unidades recordadas y varía entre 0 y 75 .

Fuente: elaboración propia.

\section{FiguRA 1}

Medias estimadas de la memoria declarativa a corto (MCP), (izquierda) y a mediano plazo (MMP) (derecha) de las unidades de textos, en los hombres (gris claro) y en las mujeres (gris oscuro), dependiendo de dosis bajas de alcohol.
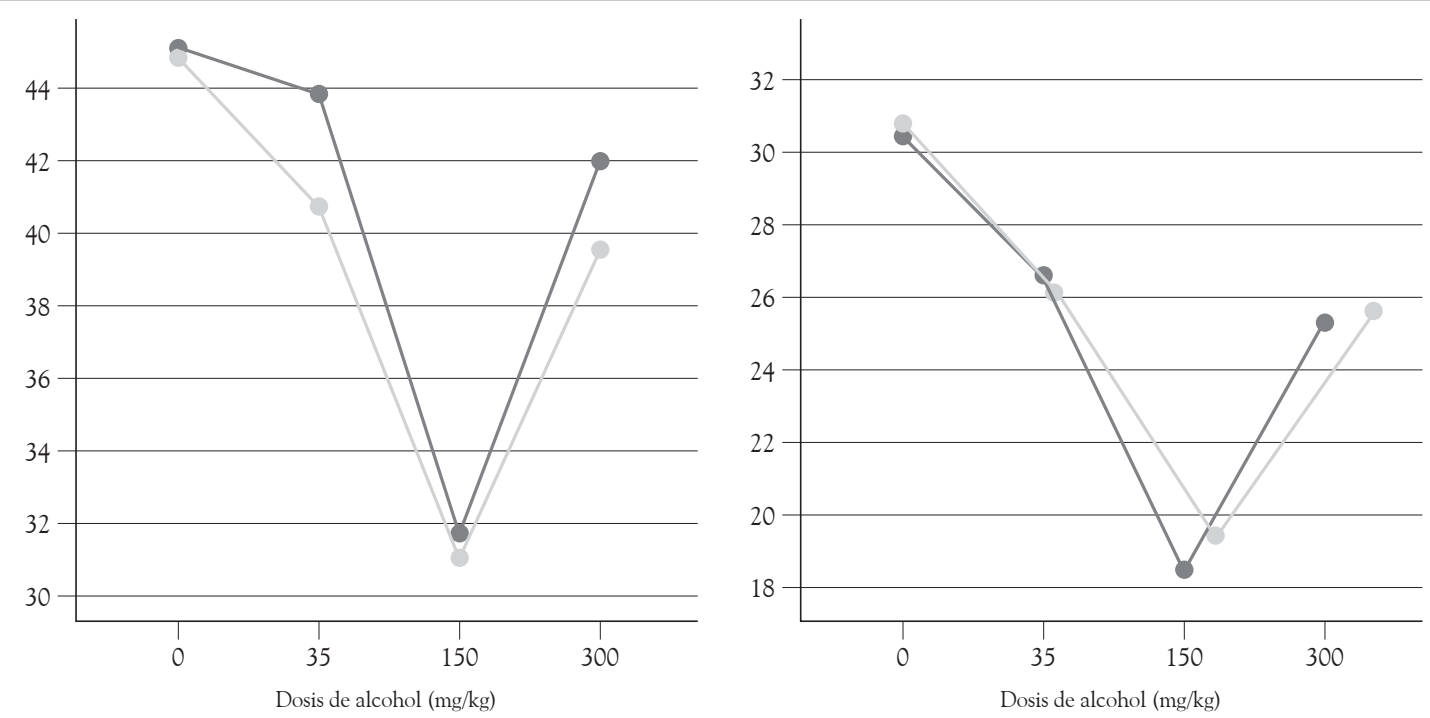

Fuente: elaboración propia.

\section{TABLA 4}

Descriptivos de la memoria declarativa, a corto y mediano plazo, de los temas de textos, según el sexo y la dosis

\begin{tabular}{ccccccccccc}
\hline \multirow{2}{*}{ Sexo } & \multicolumn{9}{c}{ Dosis de alcohol } \\
\cline { 3 - 10 } & & \multicolumn{2}{c}{0} & \multicolumn{2}{c}{35} & \multicolumn{2}{c}{150} & \multicolumn{2}{c}{300} \\
\hline \multirow{2}{*}{ Hombres } & Media & DT & Media & DT & Media & DT & Media & DT \\
& MCP & 16.63 & 3.99 & 15.25 & 4.43 & 11.5 & 3.7 & 12.38 & 2.61 \\
& MMP & 11.63 & 1.59 & 11 & 2 & 7.88 & 2.29 & 7.38 & 2.38 \\
& Mujeres & 17.38 & 1.84 & 16.38 & 2.13 & 12.63 & 2.92 & 13.38 & 2.61 \\
& MMP & 11.83 & 1.92 & 11.13 & 1.35 & 7.25 & 2.71 & 8.75 & 2.81 \\
\hline
\end{tabular}

Nota. $N=64$. La escala de medida hace referencia al número de temas recordados y varía entre 0 y 21 para la MCP.

Fuente: elaboración propia. 
FIGURA 2

Medias estimadas de la memoria declarativa a corto (MCP), (izquierda) y a mediano plazo (MMP) (derecha) de los temas de los textos, en los hombres (gris claro) y en las mujeres (gris oscuro), dependiendo de dosis bajas de alcohol.
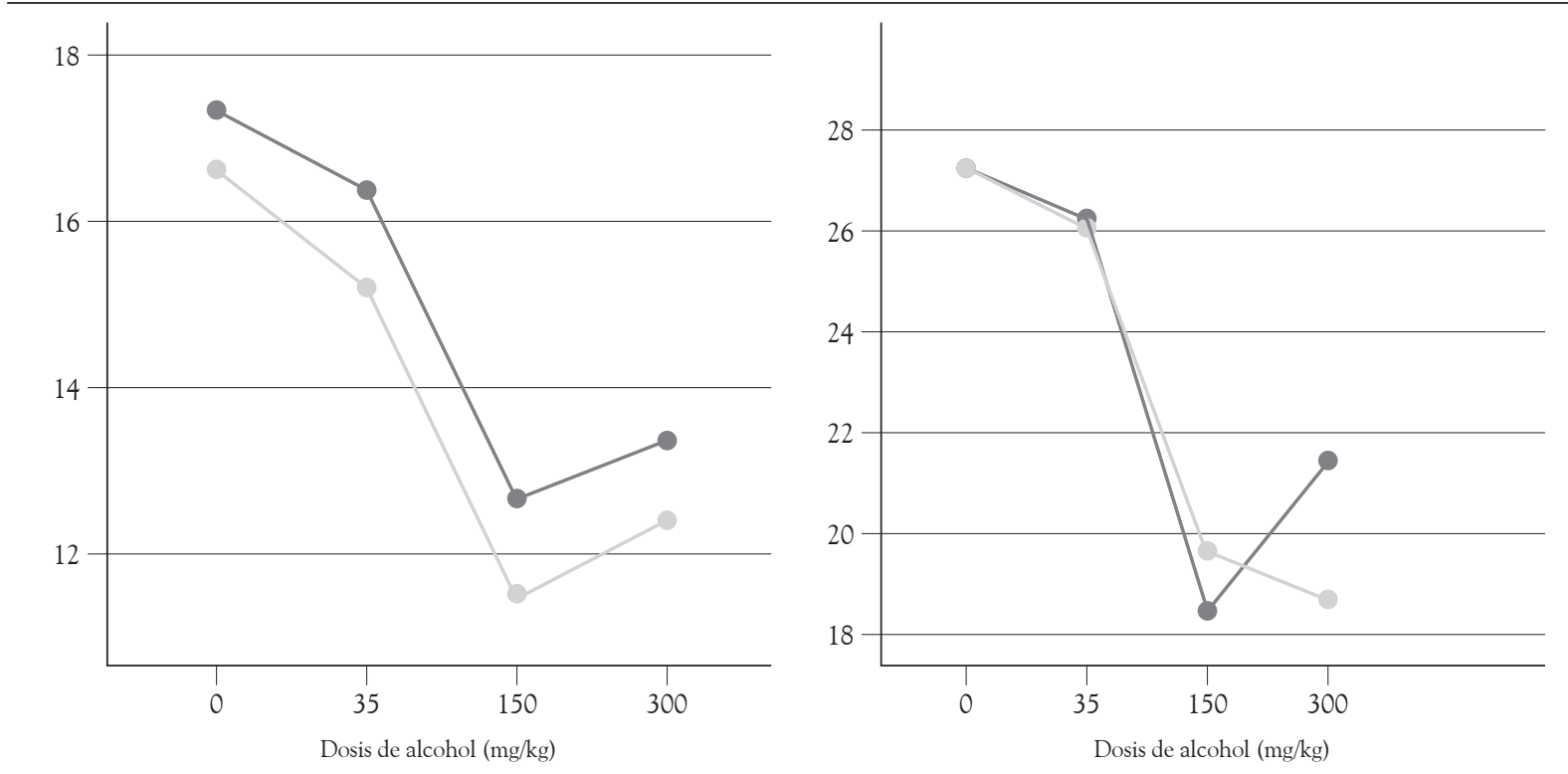

Fuente: elaboración propia.

\section{Efectos del sexo}

Se observa una diferencia mínima, no significativa, entre sexos en la MCP $\left(\eta^{2}=0.024\right)$ y su disminución hasta desaparecer en la medida en que pasa el tiempo, en la MMP $\left(\eta^{2}=0.000\right)$, como lo muestran los tamaños del efecto en la Tabla 5.

TABLA 5

ANOVA de los efectos del sexo sobre el recuerdo a corto y a mediano plazo de la memoria declarativa de unidades de textos

\begin{tabular}{|c|c|c|c|c|c|c|c|c|}
\hline Origen & & $\mathrm{SC}$ & $g l$ & $\mathrm{MC}$ & F & $p$ & $\eta^{2}$ & Potencia \\
\hline Sexo & \multirow{2}{*}{$\mathrm{MCP}$} & 42.25 & 1 & 42.25 & \multirow{2}{*}{0.340} & \multirow{2}{*}{0.569} & \multirow{2}{*}{0.024} & \multirow{2}{*}{0.085} \\
\hline Error & & 1739.18 & 14 & 124.22 & & & & \\
\hline Sexo & \multirow{2}{*}{ MMP } & 1.000 & 1 & 1.000 & \multirow{2}{*}{0.006} & \multirow{2}{*}{0.937} & \multirow{2}{*}{0.000} & \multirow{2}{*}{0.051} \\
\hline Error & & 2166.500 & 14 & 154.750 & & & & \\
\hline
\end{tabular}

Nota: $N=64 \cdot \eta^{2}:$ tamaño del efecto.

Fuente: elaboración propia.
Las Tablas 5 y 6 presentan los resultados del recuerdo de los temas de los textos bajo el efecto de cada una de las dosis. Como en el recuerdo de las unidades, el recuerdo de los temas a mediano plazo $\left(\eta^{2}=0.005\right)$ también fue inferior al recuerdo de los mismos a corto plazo $\left(\eta^{2}=0.071\right)$. 
TABLA 6

ANOVA de los efectos del sexo sobre el recuerdo a corto y a mediano plazo de la memoria declarativa de los temas de textos

\begin{tabular}{|c|c|c|c|c|c|c|c|c|}
\hline Origen & & SC & $g l$ & $\mathrm{MC}$ & $F$ & $p$ & $\eta^{2}$ & Potencia \\
\hline Sexo & \multirow{2}{*}{ MCP } & 16.0 & 1 & 16.0 & \multirow{2}{*}{1.076} & \multirow{2}{*}{0.317} & \multirow{2}{*}{0.071} & \multirow{2}{*}{0.162} \\
\hline Error & & 208.25 & 14 & 14.87 & & & & \\
\hline Sexo & \multirow{2}{*}{ MMP } & 0.76 & 1 & 0.76 & \multirow{2}{*}{0.072} & \multirow{2}{*}{0.792} & \multirow{2}{*}{0.005} & \multirow{2}{*}{0.057} \\
\hline Error & & 148.09 & 14 & 10.57 & & & & \\
\hline
\end{tabular}

$N=64$

Fuente: elaboración propia.

Los resultados presentados hasta ahora, $1^{\circ}$ ) no muestran diferencias estadísticamente significativas entre los sexos en el recuerdo a corto plazo ni en el recuerdo a mediano plazo de unidades ni de temas, como consecuencia del consumo agudo de dosis bajas de alcohol y $2^{\circ}$ ) el recuerdo a mediano plazo, tanto de unidades como de temas, fue menor que el recuerdo a corto plazo, en ambos sexos.

En relación con el efecto de las dosis de alcohol, estas produjeron diferencias significativas ( $p$ $<0.01)$ a corto y a mediano plazo, siendo mayor su efecto a mediano plazo $\left(\eta^{2}=0.561\right)$, como se puede observar en la Tabla 7 .
Estos resultados permiten rechazar la $\mathrm{H}_{0}$ de igualdad de efectos de las dosis bajas sobre la memoria declarativa con un $\alpha \leq 0.01$ en el recuerdo de eventos con un tamaño del efecto entre 0.404 y 0.561 , tanto a corto como a mediano plazo.

En relación con el recuerdo de los temas, la Tabla 8 muestra unos resultados que también permiten rechazar la $\mathrm{H}_{0}$ de igualdad de los efectos de las dosis sobre el recuerdo de temas, con un $\alpha \leq 0.01$ y un tamaño del efecto entre 0.439 y 0.629 , tanto a corto como a mediano plazo.

\section{Efectos de las dosis}

\section{TABLA 7}

ANOVA mixto de los efectos de las dosis bajas de alcohol sobre el recuerdo a corto (MCP) y mediano plazo MMP) de unidades de textos

\begin{tabular}{|c|c|c|c|c|c|c|c|c|}
\hline Fuente & & $\mathrm{SC}$ & $g l$ & $\mathrm{MC}$ & F & $p$ & $\eta^{2}$ & Pot. \\
\hline Dosis & & 1700.56 & 3 & 566.85 & 9.48 & $0.000 * * *$ & 0.404 & 0.995 \\
\hline Dosis*Sexo & $\mathrm{MCP}$ & 23.62 & 3 & 7.87 & 0.13 & 0.941 & 0.009 & 0.072 \\
\hline Error & & 2509.31 & 42 & 59.74 & & & & \\
\hline Dosis & & 1084.37 & 3 & 361.45 & 17.876 & $0.000 * * *$ & 0.561 & 1.000 \\
\hline Dosis*sexo & MMP & 3.87 & 3 & 1.292 & 0.064 & 0.979 & 0.005 & 0.060 \\
\hline Error & & 849.25 & 42 & 20.22 & & & & \\
\hline
\end{tabular}

$N=64$

Fuente: elaboración propia. 
TABLA 8

ANOVA mixto de los efectos de las dosis bajas de alcohol sobre el recuerdo a corto (MCP) y mediano plazo MMP) de temas de textos

\begin{tabular}{|c|c|c|c|c|c|c|c|c|}
\hline \multicolumn{2}{|c|}{ Fuente } & SC & $g l$ & $\mathrm{MC}$ & F & $p$ & $\eta^{2}$ & Potencia \\
\hline Dosis & & 264.62 & 3 & 88.20 & 10.94 & $0.000 * * *$ & 0.439 & 0.998 \\
\hline Dosis*sexo & MCP & 0.37 & 3 & 0.12 & 0.016 & 0.997 & 0.001 & 0.052 \\
\hline Error & & 338.5 & 42 & 8.06 & & & & \\
\hline Dosis & & 204.04 & 3 & 68.01 & 23.75 & 0.000 & 0.629 & 1.0 \\
\hline Dosis*sexo & MMP & 8.42 & 3 & 2.80 & 0.980 & 0.411 & 0.065 & 0.248 \\
\hline Error & & 120.28 & 42 & 2.86 & & & & \\
\hline
\end{tabular}

$N=64$

Fuente: elaboración propia.

Las diferencias de los efectos provocados por las dosis se muestran en la Tabla 9. En el recuerdo a corto plazo la mayor diferencia se produjo por la dosis de $150 \mathrm{mg}$, que redujo significativamente la memoria declarativa, en comparación con las demás dosis, con un $\alpha \leq 0.01$ en todos los casos (véanse Figuras 1 y 2, Tabla 9a). Resultados similares ocurrieron en la memoria a mediano plazo, donde, además, la dosis de 300 mg produjo efectos estadísticamente significativos en comparación con el control $(\alpha \leq 0.01)$ y la dosis de $150 \mathrm{mg}(\alpha$ $\leq 0.05)$.

TABLA 9

Comparaciones por pares de dosis de alcohol. Memoria declarativa a corto plazo. Unidades de textos

\begin{tabular}{|c|c|c|c|c|c|c|}
\hline \multicolumn{7}{|c|}{ Recuerdo a corto plazo } \\
\hline \multirow{2}{*}{ (I) Dosis } & \multirow{2}{*}{ (J) Dosis } & \multirow{2}{*}{ Dif. de medias $(\mathrm{I}-\mathrm{J})$} & \multirow{2}{*}{ ET } & \multirow{2}{*}{$p$} & \multicolumn{2}{|c|}{ IC $95 \%$} \\
\hline & & & & & Lím. Inf. & Lím. Sup. \\
\hline \multirow{3}{*}{ Control } & 35 & 2.68 & 2.79 & 0.352 & -3.30 & 8.67 \\
\hline & 150 & 13.68 & 2.50 & $0.000 * * *$ & 8.31 & 19.06 \\
\hline & 300 & 4.25 & 2.51 & 0.112 & -1.13 & 9.63 \\
\hline \multirow{2}{*}{35} & 150 & 11.0 & 2.84 & $0.002 * *$ & 4.89 & 17.10 \\
\hline & 300 & 1.56 & 2.81 & 0.588 & -4.47 & 7.60 \\
\hline 150 & 300 & -9.43 & 2.89 & $0.006 * *$ & -15.65 & -3.22 \\
\hline \multicolumn{7}{|c|}{ Recuerdo a mediano plazo } \\
\hline \multirow{3}{*}{ Control } & 35 & 4.18 & 1.75 & $0.031 *$ & 0.43 & 7.94 \\
\hline & 150 & 11.5 & 1.23 & $0.000 * * *$ & 8.85 & 14.14 \\
\hline & 300 & 5.06 & 1.52 & $0.005 *$ & 1.78 & 8.33 \\
\hline \multirow{2}{*}{35} & 150 & 7.31 & 1.59 & $0.000 * * *$ & 3.88 & 10.73 \\
\hline & 300 & 0.87 & 1.63 & 0.602 & -2.64 & 4.39 \\
\hline 150 & 300 & -6.43 & 1.73 & $0.002 *$ & -10.15 & -2.72 \\
\hline
\end{tabular}

Nota: $N=64$. ET: Error típico de la diferencia de medias.

Fuente: elaboración propia. 
Por último, la Figura 3 ilustra las diferencias de los efectos del sexo y de las dosis sobre la memoria declarativa, en términos de comparaciones entre los tamaños de los efectos. Se observa que las diferencias entre los sexos no generaron efectos sobre la memoria declarativa, y que fueron las dosis de alcohol las que provocaron las diferencias observadas en el recuerdo de las unidades y de los temas de los textos utilizados.

\section{Discusión}

Esta investigación se realizó con los supuestos de que las dosis, aunque bajas, producirían diferencias significativas en los 2 componentes de la memoria declarativa, episódica y semántica, tanto a corto como a mediano plazo, y que estos efectos no serían iguales en los 2 sexos. El supuesto de los efectos diferenciales generados por las dosis se fundamentó en la literatura consultada, que reporta que la administración aguda del alcohol, aun en dosis bajas, altera principalmente la memoria episódica y luego la semántica, dejando casi inalterada el recuerdo señalado por claves asociadas, según la revisión realizada por Söderlund, Parker, Schwartz y Tulving (2005). El supuesto de las diferencias producidas por el sexo se fundamentó en los efectos diferentes observados en los 2 sexos, debidos a las diferencias en la absorción, distribución y metabolismo del alcohol en los organismos masculino y femenino. Los resultados no mostraron diferencias significativas por sexo, aunque a corto plazo los hombres se vieron ligeramente más afectados que las mujeres en ambos componentes del recuerdo explícito o declarativo, como se ilustra en las figuras correspondientes. En el recuerdo a mediano plazo, no se observaron diferencias, aunque bajo $300 \mathrm{mg}$ se presentó una muy leve interacción, no significativa, entre el sexo y la dosis (Figura 2, derecha). La dosis de alcohol sí generó diferencias significativas a corto y a mediano plazo, pero especialmente a mediano plazo; y, particularmente, la de $150 \mathrm{mg}$, cuyos efectos se diferenciaron en todas las combinaciones: 150 vs. control, 150 vs. 35 y 150 vs. 300, observándose mayor deterioro del recuerdo en comparación con el control, con 150 y un poco menor, estos efectos se comparan con los producidos por $300 \mathrm{mg}$ (Tabla 9). Estos resultados son similares solo parcialmente con los reportados en la literatura. En este estudio los efectos más perjudiciales se hallaron en el recuerdo de los temas, el componente semántico, no en el recuerdo de

\section{Figura 3}

Tamaños del efecto $\left(\eta^{2}\right)$ entre las MCP y MMP de las Unidades y de los Temas de los textos estudiados, por sexo y por dosis

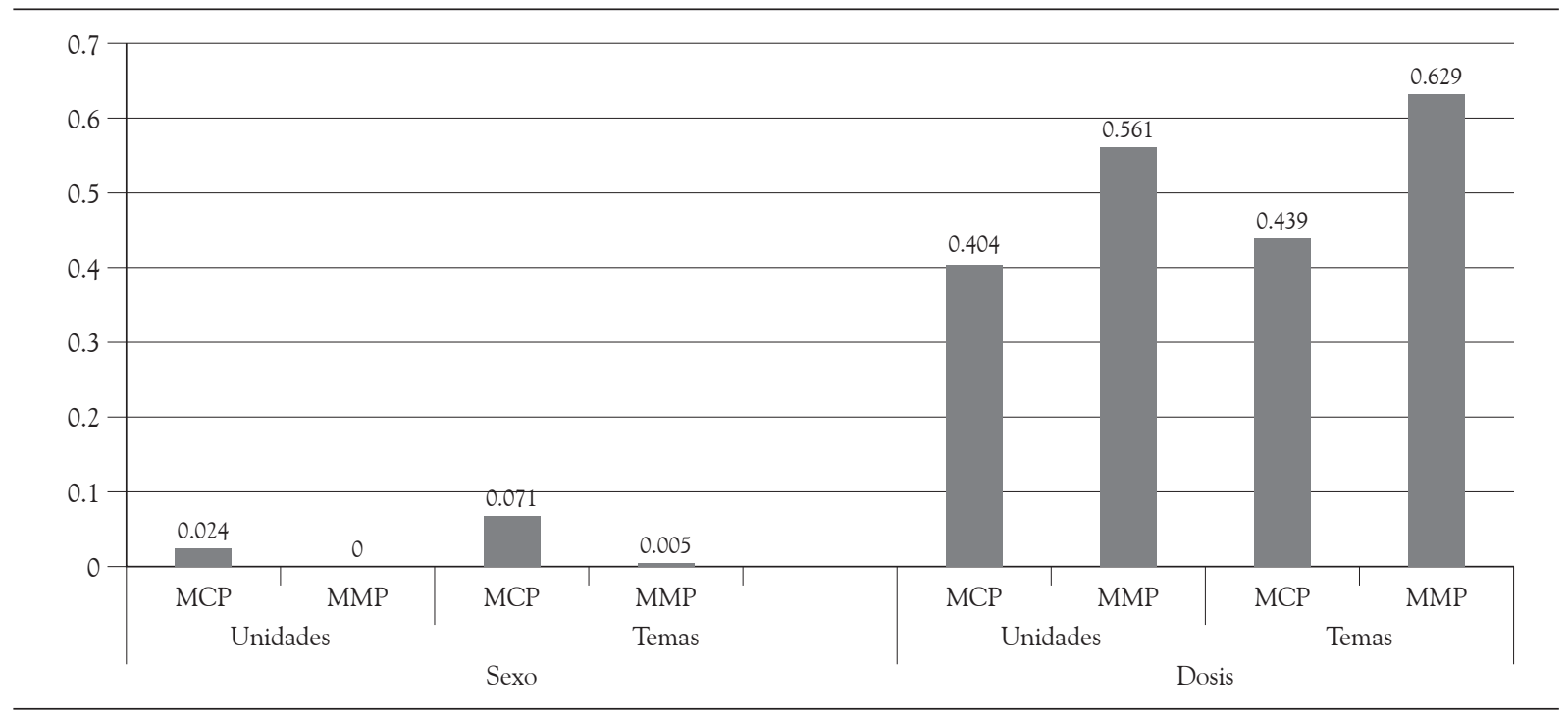

Fuente: elaboración propia. 
los episodios, las unidades de los textos. Los resultados son similares en relación con el tiempo de consolidación: el alcohol afectó más el recuerdo en el de mediano que en el de corto plazo, en ambos componentes de la memoria declarativa. Esta confirmación de resultados permite suponer que el alcohol posiblemente afecta la fase de consolidación de la memoria declarativa, porque altera la trascripción génica o la síntesis de proteínas o por algún subproceso de estos mecanismos de la memoria. Dado que el procedimiento de aplicación de las pruebas se realizó de manera estandarizada, en cuanto al tiempo de aplicación, voces, iluminación, presencia de un único investigador, ausencia de registro durante la aplicación experimental, observación y registro de los datos, que fueron realizados por personas diferentes a los investigadores y a sus asistentes, es poco probable la presencia de factores que hubieran alterado de forma relevante la validez interna de los resultados, aunque sí es posible que se hayan presentado imprecisiones en la observación o en el registro, por haber sido realizadas por personas que no participaron en las otras partes del estudio. Con todo, las diferencias individuales pudieron servir como factor de confusión. Se espera realizar un análisis intra del factor sujetos, que no se ha tenido en cuenta todavía en un examen más detallado de los resultados.

Las diferencias en los resultados de otros estudios en relación con los componentes de la memoria declarativa, pueden deberse a diferencias en la estructuración de las tareas solicitadas a los participantes. Este estudio utilizó la edición WMS-III de 2004, una versión ya diseñada cuando se estaban publicando los efectos diferentes del alcohol sobre la memoria episódica y la semántica. Tal vez, "unidades" y "temas" de los textos del WMS-III no contengan indicadores suficientemente discriminatorios de los componentes episódicos y semánticos de la memoria explícita. Quizá sea necesario diseñar pruebas más específicas para la evaluación de estos procesos.

\section{Referencias}

Baddeley, A. (2000). The Episodic Buffer: A new component of working memory. Trends in Cognitive Sciences, 4, 417-423.

Blitzer, R. D., Gil, O. \& Landau, E. M. (1990). Longterm potentiation in rat hippocampus is inhibited by low concentrations of ethanol. Brain Research, 537, 203-208.

Eichenbaum, H. (2001). A cortical-hippocampal system for declarative memory. Neuroscience, 1, 41-50.

Kandel, E. R. (2001). The molecular biology of memory storage: A dialogue between genes and synapses. Science, 294, 1030-1038.

Kirchner, T. R. \& Sayette, M. A. (2002). Effects of alcohol on controlled and automatic memory processes. Experimental and Clinical Psychopharmachology, 2, 167- 175.

Matthews, D. B. \& Silvers, J. R. (2004). The use of acute ethanol administration as a tool to investigate multiple memory systems. Neurobiology of Learning and Memory, 82, 299-308.

Ray, S., Bates, M. E. \& Bly, B. M. (2004). Alcohol's dissociation of implicit and explicit memory processes: Implications of a parallel distributed processing model of semantic priming. Experimental and Clinical Psychopharmacology, 12, 118-125.

Squire, L. R. \& Zola, S. M. (1996). Structure and function of declarative and nondeclarative memory systems. Proceedings of the National Academy of Sciences, 93, 13515-13522.

Söderlund, H., Parker, E. S., Schwartz, B. L. \& Tulving, E. (2005). Memory encoding and retrieval on the ascending and descending limbs of the blood alcohol concentration curve. Psychopharmacology, 182 (2), 305-317. DOI: 10.1007/s00213-005-0096-2

Suzuki, A., Josselyn, S. A., Frankland, P. W., Masushige, A., Silva, A. J. \& Kida, S. (2004). Memory reconsolidation and extinction have distinct temporal and biochemical signatures. Journal of Neuroscience, 24, 4787-4795.

Tulving, E. (2002). Episodic memory: From mind to brain. Annual Review of Psychology, 53, 1-25. 
Wallner, M., Hanchar, H. J. \& Olsen, R. W. (2006). Low dose acute alcohol effects on $\mathrm{GABA}_{\mathrm{A}}$ receptor subtypes. Pharmachology and Therapeutics, 112, 513-528.

Wan, F. J., Berton, F., Madamba, S. G., Francesconi, W. \& Siggins, G. R. (1996). Low ethanol concentrations enhance GABAergic inhibitory postsynaptic potentials in hyppocampal pyramidal neurons only after block of $\mathrm{GABA}_{\mathrm{B}}$ receptors. Proceedings of the National Academy of Sciences, 93, 5049-5054.
Wang, S. -H. \& Morris, R. G. M. (2010). Hippocampalneocortical interaction in memory formation, consolidation, and reconsolidation. Annual Review of Psychology, 61, 49-79.

Wechsler, D. (2004). Manual teórico, WMS-III. Escala de memoria de Wechsler-III. Barcelona: TEA Ediciones S.A.

Zwerling, C. \& Jones, M. P. (1999). Evaluation of the effectiveness of low doses of blood alcohol concentration laws for younger drivers. American Journal of Preventive Medicine, 16, 76-80. 
